\title{
Experimental Characterization of Non-Stationary V2I Radio Channel in Tunnels
}

\author{
Marwan Yusuf, Emmeric Tanghe, Luc Martens, \\ and Wout Joseph \\ Department of Information Technology \\ IMEC-WAVES, Ghent University \\ Ghent, Belgium \\ marwan.yusuf@ugent.be
}

\author{
Frederic Challita, Pierre Laly, Davy P. Gaillot, \\ and Martine Liénard \\ Electronics Department \\ IEMN-TELICE, University of Lille \\ Lille, France
}

\begin{abstract}
The fading process in vehicular communications is inherently non-stationary. In this paper, vehicle-to-infrastructure (V2I) radio channel measurements are performed inside a tunnel for low and medium traffic conditions to estimate the stationarity time, in addition to the time-varying RMS delay and Doppler spreads. Furthermore, we show the good fit of the spreads to a lognormal distribution, as well as for the Rician K-factor of the fading amplitude. From our analysis we conclude that the traffic density has an impact on the large-scale parameters as it increases delay and Doppler spreads, while reducing the correlation between them as well as the average $K$-factor. Larger traffic densities may be required to impact the stationarity time.
\end{abstract}

Index Terms-vehicular, propagation, measurement, delay, Doppler, correlation, tunnel, stationarity

\section{INTRODUCTION}

A $\mathrm{N}$ essential requirement for the development of vehicular systems is the accurate modeling of the propagation channel in different scenarios and environments [1]. One of the unexplored environments that needs more attention is tunnels. Being a confined environment, propagation behavior in tunnels differs from other environments as it plays the role of an oversized waveguide [2]. Deterministic channel models for tunnels include: waveguide models, ray tracing models and numerical methods for solving Maxwell's equations in tunnel environments [3]. These methods suffer from large computational complexity and incomplete description of the propagation environment (scatterers, mobility, traffic, etc.). On the other hand, empirical stochastic models that are obtained from measurements in real traffic condition describe the specific environment with less computational cost [3]. As the propagation is influenced by many factors (e.g. tunnel geometry, material, obstacles, Tx-Rx setup, traffic), more practical measurements are required to characterize and model the propagation in tunnels.

One of the main challenges for vehicular communications is the rapidly time-varying radio propagation channel. As a result, the widely used assumption of wide-sense stationarity (WSS) uncorrelated scattering (US) channel is no longer satisfied [4], [5]. The non-stationary fading process of vehicular

This research was supported by the VLAIO project "Smart Highway" and the EOS project "Multi-service Wireless Network (MUSE-WINET)". channels can be characterized by assuming local stationarity for a finite region in time and frequency. The author in [5] provides a theoretical framework that extends the scattering function of the WSSUS to a time-frequency (TF) dependent local scattering function (LSF). Based on the LSF, power profiles of delay (PDP) and Doppler (DPP) can be estimated, and subsequent analysis of the corresponding second central moments can be performed. In addition, the channel correlation function $(\mathrm{CCF})$, which describes the correlation of scatterers seperated by lags in the time, frequency, delay and Doppler domains [5], can be used to estimate the stationarity time based on realistic measurements [6].

Several measurements results have been published that investigate propagation in tunnels. Some of these studies investigate propagation in subway tunnels [7], where the geometry and traffic conditions are different from road tunnels. Others investigate tunnels in terms of only path loss [8]. Authors in [9] study the field distribution in the transverse plane and the correlation in both transverse and longitudinal directions. These studies investigate propagation under no traffic conditions and do not include dispersion parameters like delay spread. On the other hand, the work in [10] suggests that a single-slope model is more adequate for the path loss when the traffic is heavy. Delay spread is measured in [11], [12] and compared to simulation results, but no statistical models are presented. In addition, the stationarity time, delay and Doppler spreads are evaluated in [13] for V2V in an empty tunnel, where a lognormal model is used to fit their statistical distribution. Similar results of the delay spread are reported in [14] for a small arched tunnel.

In this paper, we characterize a non-stationary V2I channel measured in a tunnel in Antwerp, Belgium, in the frequency band of LTE-V radio interface [15]. We investigate the stationarity time based on the CCF introduced in [5], and statistically model the delay and Doppler spreads in the channel across stationarity regions. Finally, we model the small-scale fading of the channel, and give indications on the impact of traffic conditions. The outline of the paper is as follows. The description of measurements and methodology of analysis are presented in Section II. Section III describes the results and discussions, while conclusions are drawn in Section IV. 


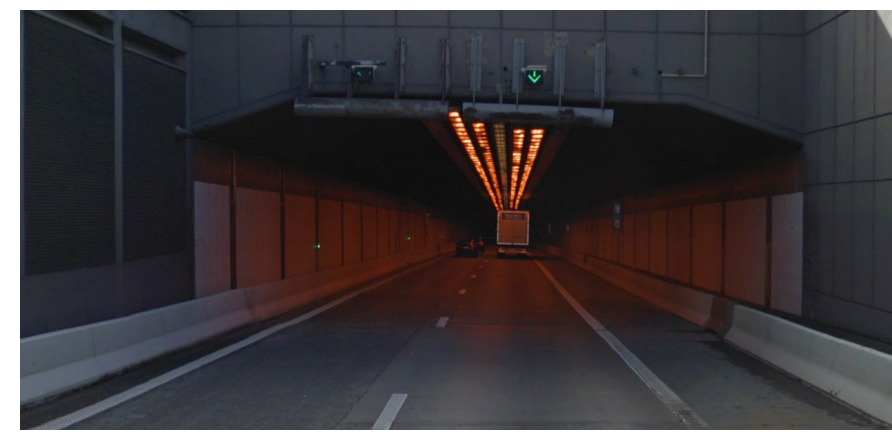

Fig. 1. Entrance of the Beveren Tunnel in Antwerp

TABLE I

MIMOSA CHANNEL SOUNDER CONFIGURATION

\begin{tabular}{lr} 
Parameter & Setting \\
\hline bandwidth & $80 \mathrm{MHz}$ \\
center frequency & $1.35 \mathrm{GHz}$ \\
Tx and Rx polarization & vertical \\
Tx and Rx antennas & omni $0.8-6.0 \mathrm{GHz}$ \\
OFDM symbol duration $T_{S}$ & $81.92 \mu \mathrm{s}$ \\
cyclic prefix duration $T_{C P}$ & $0 \leq T_{C P} \leq T_{S}$ \\
channel acquisition time & $2\left(T_{S}+T_{C P}\right) \leq 327.68 \mu \mathrm{s}$ \\
total recording time per trip & $54 \mathrm{~s}$ \\
\hline
\end{tabular}

\section{Description of MeAsurements And METHODOLOGY}

\section{A. Measurement setup and scenario}

Channel measurements are performed with the MIMOSA radio channel sounder [16]. We use $80 \mathrm{MHz}$ of transmission bandwidth centered around a carrier frequency of $1.35 \mathrm{GHz}$. This carrier frequency lies conveniently within the operating band of the LTE-V standard [15] radio interface that supports V2I communications (named Uu-interface), which operates in the licensed $2 \mathrm{GHz}$ band (880-2690 MHz). For this measurement campaign, a single wideband omnidirectional antenna is used at both Tx and Rx for the sounding signal, while we add 6 patch antennas at Rx just for synchronization and detection enhancement. The data from the Tx antenna is modulated onto the carrier using orthogonal frequency division multiplexing (OFDM). Table I summarizes the technical configuration of the MIMOSA channel sounder used for this measurement campaign.

Measurements have been carried out in the Beveren tunnel in Antwerp, Belgium. The one-kilometer tunnel has two rectangular tubes of $10 \times 5 \mathrm{~m}$ cross-section, with two lanes per direction, in addition to a roadside lane. Along the tunnel, there are lights, pipes and concrete blocks on the sides, as shown in Fig. 1. In order to follow the V2I scenario, the Tx antenna is placed inside around the middle of tunnel through an emergency exit door at $2 \mathrm{~m}$ height, as shown in Fig. 2. The $\mathrm{Rx}$ antenna is mounted on the rooftop of a van carrying the $\mathrm{Rx}$ inside. The van moves through the tunnel at $90 \mathrm{~km} / \mathrm{h}$ speed, crossing Tx position during a total trip of 54 seconds. Two types of trips are made: medium-traffic (MT) trip where 10 to

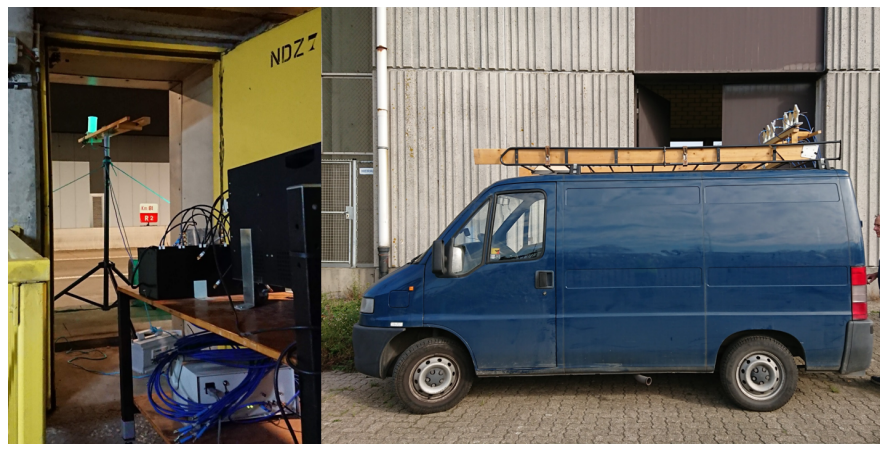

Fig. 2. Tx as a roadside unit with the antenna fixed inside the tunnel through an emergency exit (left) and the van loaded with Rx and the antenna mounted on the rooftop (right)

15 vehicles are present inside the tunnel during measurements, and low-traffic (LT) trip where the number of vehicles is less than three. Due to storage limitations of the channel sounder, we obtain a snapshot repetition time $t_{s}=975.3 \mu \mathrm{s}$. With this parameters setting, we capture a total number of snapshots $S$ $=44032$ snapshots, each with $Q=819$ samples in frequency domain, and we achieve a maximum Doppler shift of $1 / 2 t_{s}=$ $512 \mathrm{~Hz}$ and a minimum resolvable delay resolution of $12.5 \mathrm{~ns}$.

\section{B. Methodology of analysis}

Due to the high mobility of Tx, Rx and scatterers in vehicular communications, the environment is rapidly changing, and the observed fading process is non-stationary. The channel sounder provides a sampled measurement of the continuous channel transfer function (CTF) that is time-varying and frequency selective.

1) LSF Estimate: The LSF is a TF-dependent representation of the power spectrum of the observed fading process in the delay and Doppler domains $(\tau, v)$. We use the discrete version of the LSF multitaper-based estimator proposed in [5]. The 2-D tapering windows applied to the CTF are computed from the discrete prolate spheroidal sequences (DPSS) and the number of used tapers is 2 in both time and frequency domains. We estimate the LSF for consecutive regions in time, within which the channel is assumed WSSUS. A sliding window is used with an extent of $M \times N$ samples in time and frequency domains, respectively.

2) Stationarity Region: The stationarity region represents the region in time and frequency within which the LSF is highly correlated. For calculating the stationarity time, we first need to estimate the LSF assuming a minimum stationarity region. We choose the region's dimension in time domain $M=128$ samples. This needs to be validated after we calculate the stationarity time; that it is indeed larger than the assumed value. Since we are interested in the stationarity time, we include the whole bandwidth of $N=Q=819$ samples in frequency domain. The sliding time shift is selected to be half of the frame size, i.e. 64 samples. With these parameters, we obtain a LSF estimate of 12.5 ns delay resolution and $4 \mathrm{~Hz}$ Doppler resolution. Fig. 3 shows two LSF estimates at 


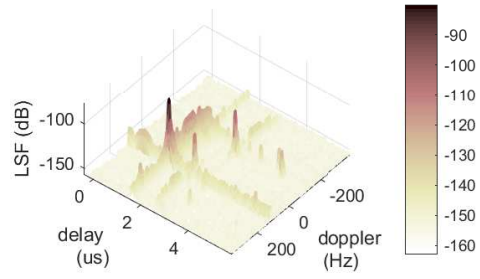

(a)

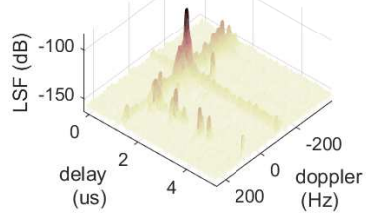

(b)
Fig. 3. LSF estimates at two instants (a) before and (b) after crossing Tx position

different instants during the trip: (a) when Rx is approaching Tx, which can be seen from the positive Doppler shift of the LOS component around $100 \mathrm{~Hz}$, and (b) after Rx crosses Tx position, resulting in the LOS Doppler shift from positive to negative $100 \mathrm{~Hz}$. Several multipath components can be observed; components from fixed scatterers are showing with less power and Doppler shifts between $+/-100 \mathrm{~Hz}$, while short-lasting components resulting from moving scatterers in both directions can reach higher positive and negative Doppler shifts.

The stationarity time can be calculated from the spread of the CCF about the origin in the Doppler lag direction [5]. We use a discrete implementation of the CCF, considering only the Doppler lag $\Delta v$ dependency for calculating the stationarity time [6]. The CCF estimate $\hat{A}$ is the 3-D DTFT of the LSF estimate $\hat{C}$

$$
\hat{A}[\Delta t, \Delta f, \Delta v]=\mathbf{F}^{3}\{\hat{C}[t, \tau, v]\}
$$

where $\Delta t, \Delta f$ and $\Delta v$ are the time lag, frequency lag and Doppler lag indexes, respectively. Then, we calculate the CCF Doppler moment as

$$
\hat{s}^{(\Delta v)}=\frac{1}{\|\hat{A}\|_{1}} \sum_{\Delta v} \sum_{\Delta t} \sum_{\Delta f}|\Delta v||\hat{A}[\Delta t, \Delta f, \Delta v]|
$$

from which the stationarity time can be evaluated as [6]

$$
T_{s}=\frac{1}{\hat{s}^{(\Delta v)}}
$$

3) Delay and Doppler Spreads: The second-order central moments of the PDP and DPP are of great importance and relevance to fading channels characterization and systems design. They have been usually assumed constant for a certain environment. However, the non-stationarity of vehicular channels allows such parameters to be defined only within a local region of stationarity. Therefore, it is reasonable to characterize the delay and Doppler spreads as time-varying channel parameters. The PDP and DPP are the projections of the LSF estimate on the delay and Doppler domains, respectively.

Based on the estimated profiles, the time-varying RMS delay and Doppler spreads can be calculated. Pre-processing is carried out separately for each stationarity region before calculating the spreads. In order to avoid spurious and noise components, we only consider components within $40 \mathrm{~dB}$ from the peak level of the estimated LSF. All components below that power threshold are set to zero [13].

4) Small-scale fading: The investigation of the small-scale amplitude is conducted in the time domain across consecutive stationarity regions. We remove the path loss and large-scale fading using a moving-average filter of the same size as the LSF estimation window. The fading distribution is acquired by calculating the CDF of the channel amplitude and comparing it to the classical fading distributions like Nakagami, Rayleigh, Weibull, and Rician [1]. This is then averaged over the whole frequency bandwidth. In order to decide on the best fitting distribution, we use the Kolmogorov-Smirnov (KS) test.

\section{Results AND Discussion}

\section{A. Stationarity Region}

As aforementioned, the stationarity time represents the region in time within which the LSF is highly correlated. The LSF correlation can be determined by the CCF spread [5]. According to (2), the CCF Doppler moment measures the Doppler correlation, which is related to the stationarity time $T_{s}$ as shown in (3). Based on our measurement data of the complete trip in the tunnel, we estimate a stationarity time $T_{s}=330 \mathrm{~ms}$. This is indeed larger than the assumed minimum value of $124.8 \mathrm{~ms}$ in Section II-B for LSF estimation. Both LT and MT trips have almost the same stationarity time, indicating that the difference in traffic density is not enough to have an impact on the stationarity.

\section{B. RMS Delay and Doppler Spreads}

For the non-stationary channel, the fading parameters can be accurately evaluated within each stationarity region, so that the channel modeling becomes physically meaningful. Based on our estimation of the stationarity time, the corresponding number of samples in time domain is $M=340$ samples. Hence, the LSF estimate is recomputed using a sliding time shift of half the stationarity region dimension. By projecting the LSF in the delay and Doppler domains, we get the PDP and DPP, respectively. Fig. 4 depicts the PDP and DPP for the central parts of the LT (upper) and MT (lower) trips inside the tunnel. The figure shows a strong reflection (i) in the first half of the tunnel in the PDP of both trips. This can be related to the metal structure at the entrance of the tunnel, visible in Fig.1. This would, indeed, have a MPC with increasing delay as Rx goes into the tunnel. In addition, the difference in traffic densities can be observed in the rich MPCs (ii) in the MT PDP compared to the LT one.

The corresponding RMS delay and Doppler spreads [6] are shown in Fig. 5. It can be seen that the spreads in the first half of the tunnel are larger compared to the second half in both trips. This can be related to the reflection coming from the entrance of the tunnel (i) in Fig. 4 (a). In addition, Fig. 5 shows that the spreads in the MT trip (b) are relatively larger than in the LT trip (a). In order to statistically characterize the spreads, we use the KS test to select the distribution by comparing the p-value of different models: lognormal, normal, Nakagami, Rayleigh, Weibull, and Rician. It is found that the lognormal 

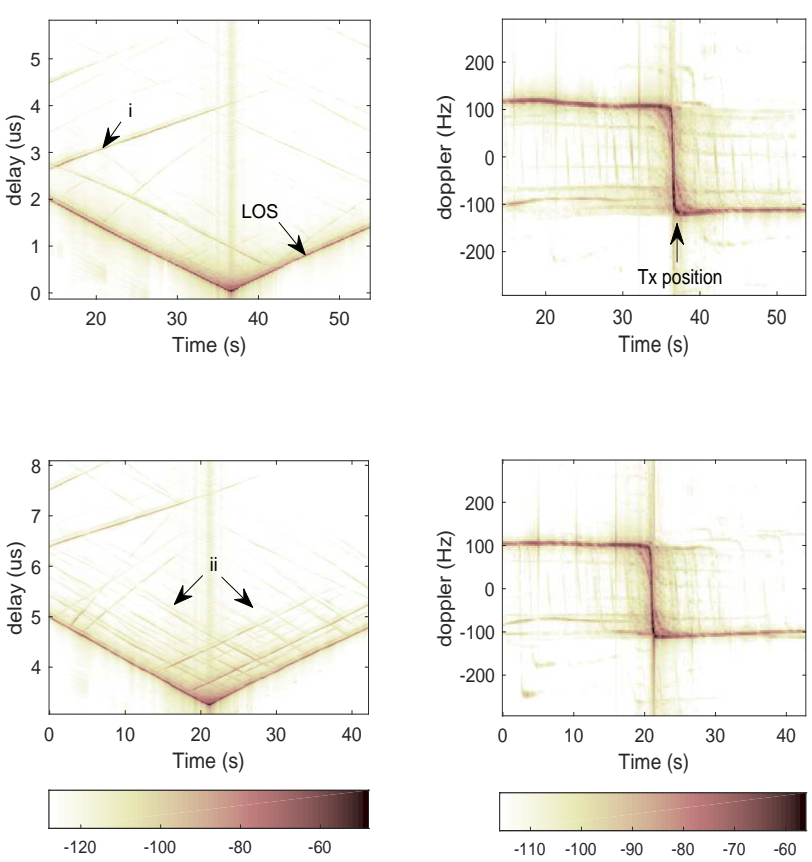

(a)

(b)

Fig. 4. Time-varying (a) PDP and (b) DPP for different trips: LT (upper) and MT (lower)

TABLE II

STATISTICS OF THE RMS DELAY AND DOPPLER SPREADS LOG-NORMAL DISTRIBUTION

\begin{tabular}{lc|cccc} 
& Trip & Mean & KS-test p-value & $\mu$ & $\sigma$ \\
\hline Delay & LT & $130.02 \mathrm{~ns}$ & 0.17 & 4.33 & 1.08 \\
& MT & $184.29 \mathrm{~ns}$ & 0.12 & 4.86 & 0.85 \\
\hline Doppler & LT & $25.08 \mathrm{~Hz}$ & 0.77 & 2.96 & 0.73 \\
& MT & $27.57 \mathrm{~Hz}$ & 0.78 & 3.18 & 0.53 \\
\hline
\end{tabular}

distribution gives the best fit to the measured parameters. Table II lists the details of the lognormal distributions for both trips, where it shows that the MT trip has slightly larger mean delay and Doppler spreads compared to the LT one.

In both trips, the delay and Doppler spreads are showing quite similar behaviors, indicating a high correlation between the two parameters. The correlation seems different in the first half of the tunnel compared to the second half, hence, the Pearson correlation coefficient is calculated separately for each half of the tunnel. Fig. 6 shows the scatter plots of the delay and Doppler spreads in log domain for both trips. It clearly shows the difference between the two halves of the tunnel in terms of parameters value as well as correlation. This is reflected in the correlation values presented in Table III, where the correlation is found to be larger in the first half compared to the second half. Again, this can be related to the MPC reflected from the tunnel entrance (i) in Fig. 4 that is only showing during the first half of the tunnel. In addition, the correlation is relatively larger in the LT trip compared to the MT one, which is expected due to the less random scatterers

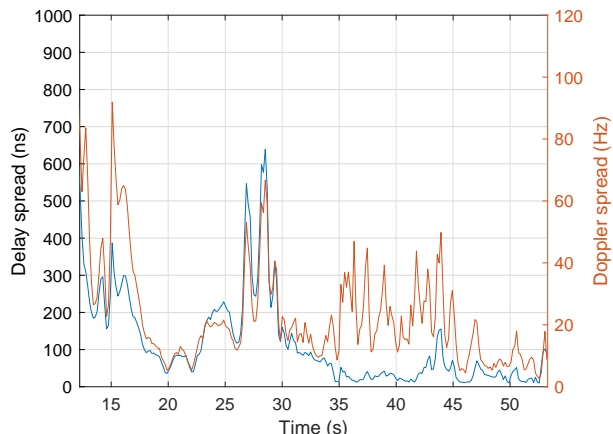

(a)

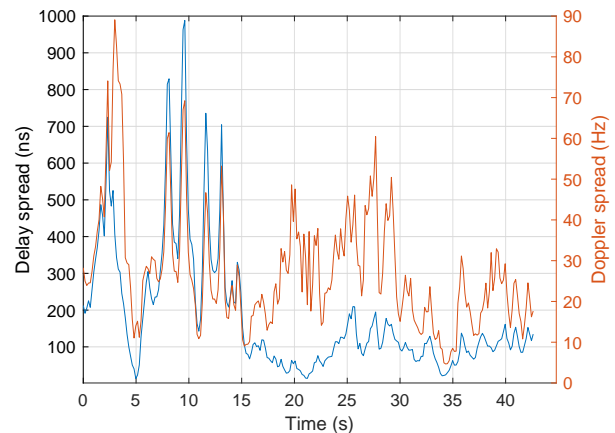

(b)

Fig. 5. RMS delay and Doppler spreads for different trips: (a) LT and (b) MT

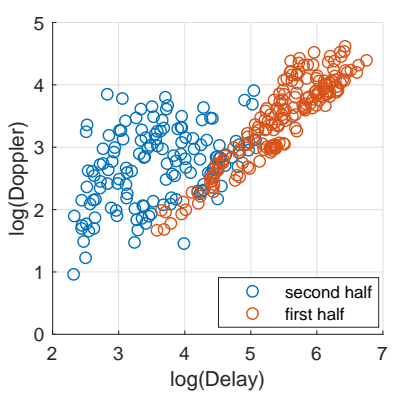

(a)

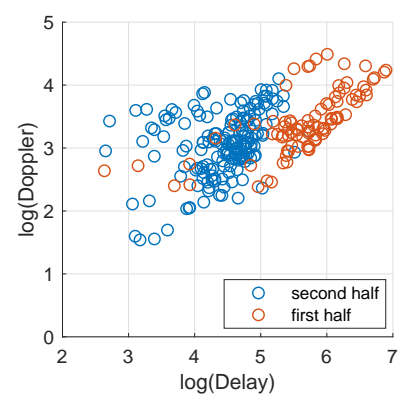

(b)
Fig. 6. Scatter plot of delay and Doppler spreads in log domain for different trips: (a) LT and (b) MT

TABLE III

PEARSON CORRELATION COEFFICIENT BETWEEN LOG(DELAY) AND LOG(DOPPLER) FOR EACH HALF OF THE TUNNEL

\begin{tabular}{c|cc} 
Trip & First half & Second half \\
\hline LT & 0.92 & 0.39 \\
\hline MT & 0.69 & 0.38 \\
\hline
\end{tabular}

(ii) in Fig. 4.

\section{Small-scale Fading Amplitude}

Table IV lists the mean p-values of the stationarity regions that passed the KS-test and the success rate of each candidate distribution for the complete trip. The Rician distribution appears to best fit the experimental results for both trips, as reported in [17], [18]. The K-factor per region is depicted 


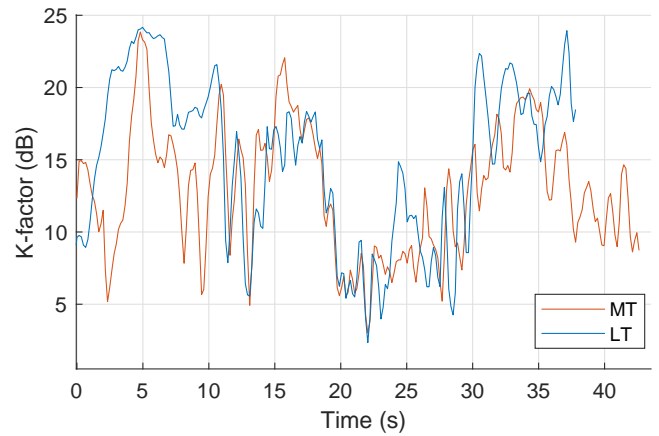

Fig. 7. K-factor of the Rician fading amplitude for both trips

TABLE IV

MEAN SIGNiFiCANCE VALUe (P) AND SUCCESS RATE (\%) OF THE KS-TEST FOR SMALL-SCALE FADING AMPLITUDE

\begin{tabular}{c|cc|cc|cc|cc}
\multirow{2}{*}{ Trip } & \multicolumn{2}{|c|}{ Rician } & \multicolumn{2}{|c|}{ Rayleigh } & \multicolumn{2}{c|}{ Nakagami } & \multicolumn{2}{c}{ Weibull } \\
& $(\mathrm{p})$ & $(\%)$ & $(\mathrm{p})$ & $(\%)$ & $(\mathrm{p})$ & $(\%)$ & $(\mathrm{p})$ & $(\%)$ \\
\hline LT & 0.57 & $\mathbf{8 5 . 6 2}$ & 0.129 & 0.35 & 0.50 & 74.63 & 0.39 & 79.61 \\
\hline MT & 0.56 & $\mathbf{8 9 . 9 5}$ & 0.064 & 0.23 & 0.49 & 76.45 & 0.41 & 87.29 \\
\hline
\end{tabular}

in Fig. 7 for the two trips, where the LT trip is shown to have a larger mean value (17.54 dB) compared to the LT one $(15.1 \mathrm{~dB})$. Using the $\mathrm{KS}$ test again with a $5 \%$ significance level against the most common distributions, the $\mathrm{K}$-factor is found to follow a lognormal distribution for the MT trip with $\mathrm{p}$-value $=0.19$. However, the LT trip did not pass the test of several candidate distributions, with the best p-value $=0.016$ for the lognormal model. Fig. 8 shows the $\mathrm{CDF}$ of the K-factor and the lognormal model parameters for the two trips.

\section{CONCLUSIONS}

Based on measurements in a tunnel in Belgium, we characterize the V2I non-stationary radio channel for low and medium traffic conditions. We apply the framework of the local scattering function and channel correlation function to characterize the stationarity time and find it to be $330 \mathrm{~ms}$ at $90 \mathrm{~km} / \mathrm{h}$ speed. We show the temporal evolution of the RMS delay and Doppler spreads, as well as the Rician K-factor of the fading amplitude. A lognormal model is found to best fit the statistical distribution of these parameters. The traffic density is found to have no effect on the stationarity time. On the other hand, it increases the delay and Doppler spreads, while reducing the correlation between them, as well as the average K-factor of the fading amplitude.

\section{REFERENCES}

[1] A. F. Molisch, F. Tufvesson, J. Karedal, and C. F. Mecklenbrauker, "A survey on vehicle-to-vehicle propagation channels," IEEE Wireless Communications, vol. 16, no. 6, pp. 12-22, 2009.

[2] D. G. Dudley, M. Lienard, S. F. Mahmoud, and P. Degauque, "Wireless propagation in tunnels," IEEE Antennas and Propagation Magazine, vol. 49, no. 2, pp. 11-26, 2007.

[3] A. Hrovat, G. Kandus, and T. Javornik, "A survey of radio propagation modeling for tunnels," IEEE Communications Surveys \& Tutorials, vol. 16, no. 2, pp. 658-669, 2014.

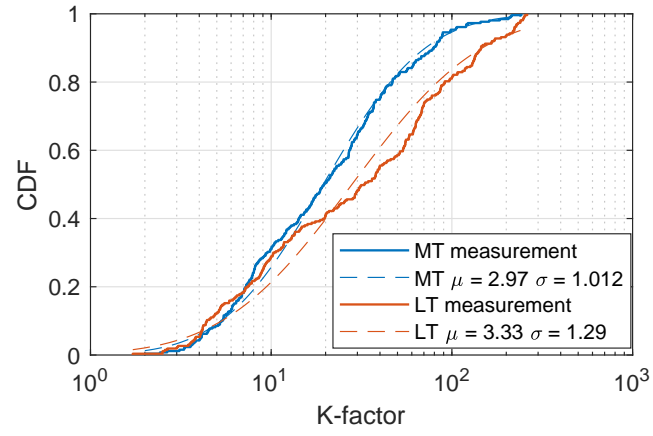

Fig. 8. CDF of the K-factor and lognormal model fit for both trips

[4] L. Bernadó, T. Zemen, F. Tufvesson, A. F. Molisch, and C. F. Mecklenbräuker, "The (in-) validity of the WSSUS assumption in vehicular radio channels." in PIMRC, 2012, pp. 1757-1762.

[5] G. Matz, "On non-WSSUS wireless fading channels," IEEE Transactions on Wireless Communications, vol. 4, no. 5, pp. 2465-2478, 2005.

[6] M. Yusuf, E. Tanghe, F. Challita, P. Laly, D. Gaillot, M. Lienard, L. Martens, and W. Joseph, "Stationarity analysis of V2I radio channel in a suburban environment," IEEE Transactions on Vehicular Technology, pp. 1-1, 2019.

[7] M. Lienard, P. Degauque, J. Baudet, and D. Degardin, "Investigation on MIMO channels in subway tunnels," IEEE Journal on Selected Areas in Communications, vol. 21, no. 3, pp. 332-339, 2003.

[8] Z. Sun and I. F. Akyildiz, "Channel modeling and analysis for wireless networks in underground mines and road tunnels," IEEE Transactions on communications, vol. 58, no. 6, 2010.

[9] J. Molina-Garcia-Pardo, M. Lienard, and P. Degauque, "Propagation in tunnels: Experimental investigations and channel modeling in a wide frequency band for MIMO applications," EURASIP Journal on Wireless Communications and Networking, vol. 2009, p. 7, 2009.

[10] M. Liénard, S. Bétrencourt, and P. Degauque, "Propagation in road tunnels: a statistical analysis of the field distribution and impact of the traffic," in Annales des télécommunications, vol. 55, no. 11-12. Springer, 2000, pp. 623-631.

[11] F. M. Pallares, F. P. Juan, and L. Juan-Llacer, "Analysis of path loss and delay spread at $900 \mathrm{mhz}$ and $2.1 \mathrm{ghz}$ while entering tunnels," IEEE Transactions on Vehicular Technology, vol. 50, no. 3, pp. 767-776, 2001.

[12] C. Garcia-Pardo, J.-M. Molina-García-Pardo, M. Lienard, D. P. Gaillot, and P. Degauque, "Double directional channel measurements in an arched tunnel and interpretation using ray tracing in a rectangular tunnel," Progress In Electromagnetics Research, vol. 22, pp. 91-107, 2012.

[13] L. Bernadó, A. Roma, A. Paier, T. Zemen, N. Czink, J. Karedal, A. Thiel, F. Tufvesson, A. F. Molisch, and C. F. Mecklenbrauker, "Intunnel vehicular radio channel characterization," in Vehicular Technology Conference (VTC Spring), 2011 IEEE 73rd. IEEE, 2011, pp. 1-5.

[14] M. Yusuf, E. Tanghe, L. Martens, P. Laly, D. P. Gaillot, M. Linard, P. Degauque, and W. Joseph, "Experimental investigation of V2I radio channel in an arched tunnel," in 2019 13th European Conference on Antennas and Propagation (EuCAP), March 2019, pp. 1-5.

[15] "Technical specification group radio access network; vehicle-toeverything (V2X) services based on LTE; user equipment (UE) radio transmission and reception. TR 36.786 v14.0.0," 3GPP, Tech. Rep., 2017.

[16] P. Laly, D. P. Gaillot, M. Liénard, P. Degauque, E. Tanghe, W. Joseph, and L. Martens, "Flexible real-time mimo channel sounder for multidimensional polarimetric parameter estimation," in Antenna Measurements \& Applications (CAMA), 2015 IEEE Conference on. IEEE, 2015, pp. $1-3$.

[17] D. Didascalou, J. Maurer, and W. Wiesbeck, "Subway tunnel guided electromagnetic wave propagation at mobile communications frequencies," IEEE Transactions on Antennas and Propagation, vol. 49, no. 11, pp. 1590-1596, 2001.

[18] S. Loredo, A. del Castillo, H. Fernández, V. M. Rodrigo-Peñarrocha, J. Reig, and L. Rubio, "Small-scale fading analysis of the vehicular-tovehicular channel inside tunnels," Wireless Communications and Mobile Computing, vol. 2017, 2017. 